\title{
Standing second sound wave in many-layer systems
}

\author{
K. Nemchenko and S. Rogova \\ Department of Physics and Energy, V.N. Karazin Kharkov National University \\ 4 Svobody Sq., Kharkov 61022, Ukraine \\ E-mail: nemchenko@bk.ru
}

Received April 19, 2011

\begin{abstract}
The variation of thermodynamic parameters of superfluid helium in standing second sound wave is studied. Such double-layer systems as heater-helium and helium-detector are considered. Heat transfer in the heater and the detector was described with usual thermal conductivity equation. Temperature and heat transfer in superfluid ${ }^{4} \mathrm{He}$ was described with the system of equations for superfluid with taking into accounts both dissipative thermal conductivity mode and second sound mode. Resonance frequency and amplitude dependence on dissipation at the heater and the detector was studied. The unusual resonance was found in the double-layer system heliumdetector.
\end{abstract}

PACS: 67.60.-g Mixtures of ${ }^{3} \mathrm{He}$ and ${ }^{4} \mathrm{He}$;

44.05.+e Analytical and numerical techniques;

43.20.Ks Standing waves, resonance, normal modes.

Keywords: superfluid helium, second sound, resonances, standing wave.

\section{Introduction}

Superfluid ${ }^{4} \mathrm{He}$ possesses a number of unique features. One of them is the existence of second sound [1]. Unlike usual sound in the second sound mode not full momentum (and pressure) fluctuates but temperature (and entropy) fluctuations take place. Second sound provides heat transfer and temperature relaxation in He II.

One of the experimental methodic to study second sound is the generation of the standing wave of second sound. This methodic was used in the first experiments dealt with second sound [2,3], as well as in the latest experiments [4], in which the possible electric properties of second sound were studied. The latter experiments resulted on theoretical consideration presented in this paper.

In this work the heat transfer model in the double-layer systems $[5,6]$ is presented. Such double-layer systems as heater-helium and helium-detector are considered. The nature of heat transfer in these layers is very different. Heat transfer in the heater and the detector is determined by dissipative process of thermal conductivity, so, it is described by usual thermal conductivity equation. Heat transfer in helium is determined by second sound and it is described by the system of equations for superfluid with taking into accounts both dissipative thermal conductivity mode and second sound mode.

\section{Double-layer system heater-helium}

At first we consider the double-layer system consisting of a heater and helium (see Fig. 1,a). The first layer is a usual substance of finite length $l_{1}$ and serves as a heater. The second layer is superfluid helium of finite length $l_{2}$. The left end of the first layer is thermally isolated. Inside the first layer heat sources are uniformly distributed.

To describe heat transfer in the first layer usual thermal conductivity equation with external uniformly distributed heat sources $q(t)$ is used

$$
\dot{T}_{1}=\chi_{1} T_{1}^{\prime \prime}+q(t), \quad q(t)=Q_{0} \cos (\omega t),
$$

where $\chi_{1}$ is the temperature conductivity of the heater, $T_{1}$ is the temperature deviation of the heater from the equilibrium value $T_{0}, Q_{0}$ is the dimensionless (divided to heat capacity) external heat sources amplitude, $\omega$ is the frequency of external heat source.
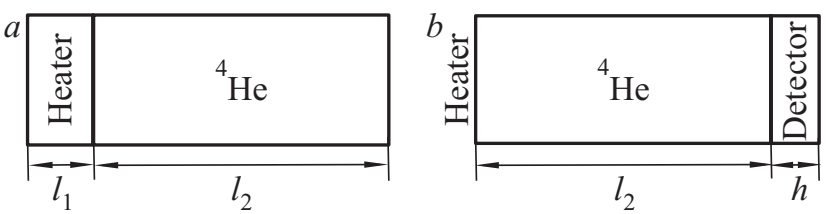

Fig. 1. The geometry of considered experiments: heater-helium (a); helium-detector (b) systems. 
At the left end the boundary condition for the heat flow in the heater has the form

$$
Q(0, t)=0
$$

and at the right end of the heater we seek the heat flow as

$$
Q\left(l_{1}, t\right)=Q_{1} \cos (\omega t+\phi),
$$

where $l_{1}$ is the length of the first layer, $Q_{1}$ and $\phi$ are parameters that are defined by equating temperatures and heat flows at the layers boundary.

Heat transfer in the second layer is described with the system of hydrodynamic equations for superfluids that takes into account both second sound and dissipation [2]

$$
\left\{\begin{array}{l}
\dot{v}_{n}+u_{2}^{2} T^{\prime}=0 \\
\dot{T}+v_{n}^{\prime}=\chi_{2} T^{\prime \prime}
\end{array}\right.
$$

Here $u_{2}$ is the second sound velocity, $v_{n}$ is the normal fluid velocity, $\chi_{2}$ is the temperature conductivity of the helium, $T$ is the temperature deviation from the equilibrium value $T_{0}$. The boundary condition at the right end of helium has the form

$$
T\left(l_{2}, t\right)=0
$$

where $l_{2}$ is the length of the second layer. The heat flow in helium is

$$
q=S T_{0} v_{n}-\kappa T^{\prime}
$$

where $S$ is the entropy of helium and $\kappa=C_{V} \chi_{2}$ is the thermal conductivity coefficient of helium. Here we should note that the second term in Eq. (6) almost at all temperatures can be neglected.

\section{Result of calculation}

To solve the problem Laplace transform was used in order to solve the problem about switching on $[7,8]$ and off the heat flow in future. Amplitude and phase of the heat flow at the boundary of the layers were obtained by equating temperatures and heat flows at the boundary.

For temperature of the heater the following expression was obtained

$$
\begin{aligned}
T_{1}(x, t)= & \frac{Q_{1} \sqrt{\sinh ^{2}\left(\lambda_{1} l_{1}\right)+\cos ^{2}\left(\lambda_{1} l_{1}\right)} \sin (\omega t-\alpha)}{\sqrt{\cosh ^{2}\left(\lambda_{1} l_{1}\right)-\cos ^{2}\left(\lambda_{1} l_{1}\right)} \lambda_{1} \chi_{1} C_{V 1}}+ \\
& +\frac{\bar{Q}_{0}}{\omega} \sin (\omega t) .
\end{aligned}
$$

Here $\lambda_{1}=\sqrt{\omega / 2 \chi_{1}}$ is the inverse thermal wavelength in the heater, $\bar{Q}_{0}=Q_{0} C_{V 1} l_{1}$ is the normalized heat flow amplitude, $\alpha=\phi-\pi / 4+\arctan \left(B_{1}(x) / A_{1}(x)\right)$ is the phase shift, and $C_{V 1}$ is the thermal capacity of the heater. Values $A_{1}(x)$ and $B_{1}(x)$ are determined by expressions

$$
\begin{aligned}
& A_{1}(x)=\cos \left(\lambda_{1} x\right) \cosh \left(\lambda_{1} x\right) \cos \left(\lambda_{1} l_{1}\right) \sinh \left(\lambda_{1} l_{1}\right)+ \\
& +\sin \left(\lambda_{1} x\right) \sinh \left(\lambda_{1} x\right) \sin \left(\lambda_{1} l_{1}\right) \cosh \left(\lambda_{1} l_{1}\right), \\
& B_{1}(x)=\sin \left(\lambda_{1} x\right) \sinh \left(\lambda_{1} x\right) \cos \left(\lambda_{1} l_{1}\right) \sinh \left(\lambda_{1} l_{1}\right)- \\
& -\cos \left(\lambda_{1} x\right) \cosh \left(\lambda_{1} x\right) \sin \left(\lambda_{1} l_{1}\right) \cosh \left(\lambda_{1} l_{1}\right) .
\end{aligned}
$$

Phase shift $\phi$ is given by

$$
\phi=-\arctan \left(\frac{\sqrt{2} k_{2} \gamma_{1}+\omega C_{V} \varsigma_{-}}{\sqrt{2} k_{1} \gamma_{2}+\omega C_{V} \varsigma_{+}}\right),
$$

and heat flow amplitude $Q_{1}$ takes the form

$$
Q_{1}=\frac{Q_{0} \xi \mu C_{V}}{\left(\gamma_{2} \cos \phi-\gamma_{1} \sin \phi\right)+\left(\gamma_{1} \cos \phi-\gamma_{2} \sin \phi\right) \mu C_{V} \omega / \sqrt{2}} .
$$

In Eqs. (7)-(10) the following expressions were used:

$$
\begin{gathered}
\gamma_{1}=\xi\left[k_{2} \cosh \left(2 k_{1} l_{2}\right)+k_{1} \cos \left(2 k_{2} l_{2}\right)\right], \\
\gamma_{2}=\xi\left[k_{1} \sinh \left(2 k_{1} l_{2}\right)-k_{2} \sin \left(2 k_{2} l_{2}\right)\right], \\
\xi=\lambda_{1} \chi_{1} C_{V_{1}}\left[\cosh ^{2}\left(\lambda_{1} l_{1}\right)-\cos ^{2}\left(\lambda_{1} l_{1}\right)\right], \\
\varsigma_{ \pm}=\mu\left[\sinh \left(2 \lambda_{1} l_{1}\right) \pm \sin \left(2 \lambda_{1} l_{1}\right)\right], \\
\mu=\cosh \left(2 k_{1} l_{2}\right)+\cos \left(2 k_{2} l_{2}\right) .
\end{gathered}
$$

Here we introduced the values $k_{1}$ and $k_{2}$, which respect to typical inverse lengths of the problem: $k_{1}$ corresponds to dissipative thermal heat wave length:

$$
k_{1}=\frac{\omega}{U} \sqrt{\frac{1}{2}\left(1-\frac{u_{2}^{2}}{U^{2}}\right)}
$$

and $k_{2}$ defines the wavelength of second sound:

$$
k_{2}=\frac{\omega}{U} \sqrt{\frac{1}{2}\left(1+\frac{u_{2}^{2}}{U^{2}}\right)} .
$$

The value $U$ determines the velocity of heat propagation:

$$
U=\sqrt[4]{u_{2}^{4}+\omega^{2} \chi^{2}}
$$

For the second layer, which is superfluid helium, the dependence on coordinate was obtained

$$
T_{2}(x, t)=-\frac{Q_{1}\left[A_{2}(x) \sin (\omega t)+B_{2}(x) \cos (\omega t)\right]}{U C_{V}\left[\cosh ^{2}\left(k_{2} l_{2}\right)-\cos ^{2}\left(k_{1} l_{2}\right)\right]} .
$$

The functions $A_{2}(x)$ and $B_{2}(x)$ are

$$
\begin{aligned}
& A_{2}(x)=\frac{U}{\omega}\left(k_{1} \cos \phi-k_{2} \sin \phi\right)\left[s_{1}(x) s_{3}\left(l_{2}\right)+s_{2}(x) s_{4}\left(l_{2}\right)\right]+ \\
& +\left(k_{2} \cos \phi+k_{1} \sin \phi\right)\left[s_{1}(x) s_{4}\left(l_{2}\right)-s_{2}(x) s_{3}\left(l_{2}\right)\right]
\end{aligned}
$$

and 
$B_{2}(x)=\frac{U}{\omega}\left(k_{1} \cos \phi-k_{2} \sin \phi\right)\left[s_{2}(x) s_{3}\left(l_{2}\right)-s_{1}(x) s_{4}\left(l_{2}\right)\right]+$ $+\left(k_{2} \cos \phi+k_{1} \sin \phi\right)\left[s_{1}(x) s_{3}\left(l_{2}\right)+s_{2}(x) s_{4}\left(l_{2}\right)\right]$.

In Eqs. (15) and (16) we used the following relations:

$$
\begin{gathered}
s_{1}(x)=\cos \left(k_{2}\left(l_{2}-x\right)\right) \sinh \left(k_{1}\left(l_{2}-x\right)\right), \\
s_{2}(x)=\sin \left(k_{2}\left(l_{2}-x\right)\right) \cosh \left(k_{1}\left(l_{2}-x\right)\right), \\
s_{3}(x)=\cos \left(k_{2} x\right) \cosh \left(k_{1} x\right), \\
s_{4}(x)=\sin \left(k_{2} x\right) \sinh \left(k_{1} x\right) .
\end{gathered}
$$

Obtained expression (14) for temperature allows describing of temperature in helium at any coordinate point inside the experimental device.

For the heat flow in helium layer we obtain the following expression:

$Q_{2}(x, t)=\frac{Q_{1}\left[A_{2 Q}(x) \sin (\omega t)+B_{2 Q}(x) \cos (\omega t)\right]}{\cosh ^{2}\left(k_{2} l_{2}\right)-\cos ^{2}\left(k_{1} l_{2}\right)}$,

where functions $A_{2 \mathrm{Q}}(x)$ and $B_{2 \mathrm{Q}}(x)$ have the form

$$
\begin{gathered}
A_{2 Q}(x)=-\left[s_{3}\left(l_{2}-x\right) s_{3}\left(l_{2}\right)+s_{4}\left(l_{2}-x\right) s_{4}\left(l_{2}\right)\right] \sin \phi- \\
-\left[s_{4}\left(l_{2}-x\right) s_{3}\left(l_{2}\right)-s_{3}\left(l_{2}-x\right) s_{4}\left(l_{2}\right)\right] \cos \phi
\end{gathered}
$$

and

$$
\begin{gathered}
B_{2 Q}(x)=\left[s_{3}\left(l_{2}-x\right) s_{3}\left(l_{2}\right)+s_{4}(x) s_{4}\left(l_{2}\right)\right] \cos \phi- \\
-\left[s_{4}\left(l_{2}-x\right) s_{3}\left(l_{2}\right)-s_{3}\left(l_{2}-x\right) s_{4}\left(l_{2}\right)\right] \sin \phi .
\end{gathered}
$$

These results allow determining of the heat flow both inside the helium and at the right boundary of the vessel with helium, i.e. in the point, where the bolometer is situated in experiments [4].

\section{The problem of thermometer measurement}

When carrying out experiments [4] temperature is measured with the help of thermometer, which is situated in the right side of the vessel with helium. Let's show that situation when thermometer measures not temperature but heat flow is possible.

Using Eq. (17) heat flow at right side of helium at $x=l_{2}$ can be written in the form

$$
Q\left(l_{2}, t\right)=Q_{2} \sin (\omega t+\beta),
$$

where phase shift is

$$
\beta=\arctan \left(\frac{s_{3} \cos \phi-s_{4} \sin \phi}{s_{4} \cos \phi-s_{3} \sin \phi}\right),
$$

and amplitude is equal to

$$
Q_{2}=\frac{Q_{1} \sqrt{s_{3}^{2}+s_{4}^{2}-2 s_{3} s_{4} \sin (2 \phi)}}{\cos \left(2 k_{2} l_{2}\right)+\cosh \left(2 k_{1} l_{2}\right)} .
$$

Let the thermometer does not change heat flow in helium. Then thermal conductivity equation for the thermome- ter $T_{t}=\chi_{B} T_{x x}$ can be written with the boundary conditions $Q_{B}(x=0)=Q_{2} \sin (\omega t+\beta), Q_{B}\left(x=l_{B}\right)=0$ (because the thermometer is heat insulated with non-conductor), where $l_{B}$ is the thermometer length, $\chi_{B}$ is the thermometer temperature conductivity.

In this case the solution of the thermal conductivity equation inside the thermometer has the form

$$
T_{B}(x, t)=Q_{2} \sin (\omega t+\beta) \frac{\cos \left(\sqrt{\omega / \chi_{B}}\left(l_{B}-x\right)\right)}{\sqrt{\chi_{B} \omega} \sin \left(\sqrt{\omega / \chi_{B}} l_{B}\right)} .
$$

From this expression we obtain the average temperature in the thermometer:

$$
\bar{T}_{B}(t)=\frac{1}{l_{B}} \int_{0}^{l_{B}} T_{B}(x, t) d x=\frac{Q\left(l_{2}, t\right)}{l_{B} \omega} .
$$

So, under definite conditions, when one side of a thermometer is thermally isolated, the thermometer will show the heat flow at its other side. In our case such thermometer will show heat flow in helium at $x=l_{2}$.

\section{Heat flow resonanses}

It is known that under boundary conditions (3) in superfluid helium temperature and heat flow resonances appear. This phenomenon was described in $[7,8]$ where experiments with given heat flow at both ends were carried out. In such case temperature and heat flow resonances are observed in all harmonics (Fig. 2,a). In this work it is shown that such problem formulation when only odd resonances
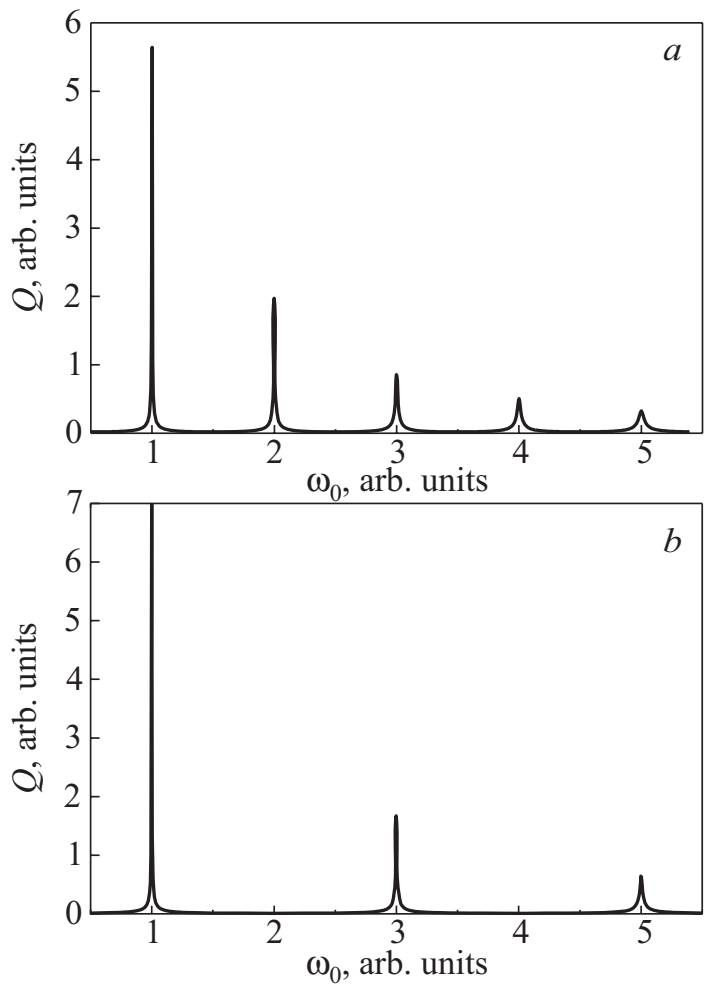

Fig. 2. Heat flow amplitude dependence on frequency excitation when at the right end is constant heat flow [7] (a) or constant temperature (Eqs. (17)-(19)) (b). 


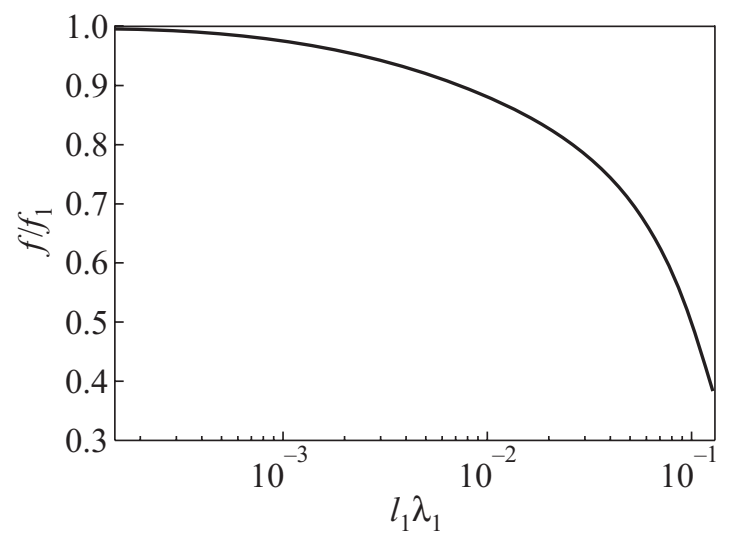

Fig. 3. First resonance dependence on the heater width.

appear is possible (Fig. 2,b). It is related with the difference in boundary conditions. In classical case right end is heat insulated $\left(Q\left(l_{2}, t\right)=0\right)$ and in our case right end is at constant temperature $\left(T\left(l_{2}, t\right)=0\right)$.

Resonance frequency dependence on length of the first layer was studied as well. It was found out that the longer the first layer is the less is resonance frequency. In Fig. 3 an example of such dependence for the first resonance is given.

\section{Double-layer system helium-detector}

Let's study the influence of the right wall of the vessel on resonances characteristics. So, we consider the right wall as a layer of $h$ width (see Fig. $1, b$ ). Heat transfer in it we describe with the usual thermal conductivity equation. Heat transfer in helium we describe with the system (4). For simplicity we consider $\chi_{2}=0$ and $l_{1}=0$. We have obtained the double-layer system (the first layer is superfluid helium and the second layer is the right wall of the vessel) with the following boundary conditions:

$$
Q(x=0, t)=Q_{0} \cos (\omega t), T(h, t)=0 .
$$

Making temperatures and heat flows equal at the boundary of the layers we obtain the following expression for temperature in superfluid helium:

$$
T(x, t)=\frac{Q_{0}\left[C_{1}(x) \sin (\omega t)+C_{2}(x) \cos (\omega t)\right]}{\alpha u_{2}\left[4 C_{V}^{2} \omega \chi \cos ^{2}\left(k l_{2}\right) b_{1}+4 \alpha^{2} u_{2}^{2} \sin ^{2}\left(k l_{2}\right) b_{2}+C_{V} \sqrt{2 \omega \chi} \alpha u_{2} \sin \left(2 k l_{2}\right) b_{3}\right]},
$$

where functions $C_{1}(x)$ and $C_{2}(x)$ are

$$
\begin{aligned}
& C_{1}(x)=4 C_{V}^{2} \omega \chi \cos \left(k l_{2}\right) \sin \left(k\left(l_{2}+x\right)\right) b_{1}-4 \alpha^{2} u_{2}^{2} \sin \left(k l_{2}\right) \cos \left(k\left(l_{2}+x\right)\right) b_{2}-C_{V} \sqrt{2 \omega \chi} \alpha u_{2} \cos \left(k\left(2 l_{2}+x\right)\right) b_{3}, \\
& C_{2}(x)=-C_{V} \sqrt{2 \omega \chi} \alpha u_{2} \cos (k x) b_{4} .
\end{aligned}
$$

The expression for the heat flow in helium is

$$
Q(x, t)=\frac{Q_{0}\left[C_{3}(x) \sin (\omega t)+C_{4}(x) \cos (\omega t)\right]}{4 C_{V}^{2} \omega \chi \cos ^{2}\left(k l_{2}\right) b_{1}+4 \alpha^{2} u_{2}^{2} \sin ^{2}\left(k l_{2}\right) b_{2}+C_{V} \sqrt{2 \omega \chi} \alpha u_{2} \sin \left(2 k l_{2}\right) b_{3}},
$$

with functions $C_{3}(x)$ and $C_{4}(x)$ :

$$
\begin{aligned}
& C_{3}(x)=4 C_{V}^{2} \omega \chi \cos \left(k l_{2}\right) \cos \left(k\left(l_{2}+x\right)\right) b_{1}+4 \alpha^{2} u_{2}^{2} \sin \left(k l_{2}\right) \sin \left(k\left(l_{2}+x\right)\right) b_{2}+C_{V} \sqrt{2 \omega \chi} \alpha u_{2} \sin \left(k\left(2 l_{2}+x\right)\right) b_{3}, \\
& C_{4}(x)=C_{V} \sqrt{2 \omega \chi} \alpha u_{2} \sin (k x) b_{4} .
\end{aligned}
$$

Hereinabove we used the following notations:

$$
\begin{aligned}
& b_{1}=\cos ^{2}(\lambda h)+\sinh ^{2}(\lambda h), b_{2}=\sin ^{2}(\lambda h)+\sinh ^{2}(\lambda h), \\
& b_{3}=\sinh (2 \lambda h)-\sin (2 \lambda h), b_{4}=\sin (2 \lambda h)+\sinh (2 \lambda h), \\
& \alpha=T_{0} C_{V 2} .
\end{aligned}
$$

Here $\lambda=\sqrt{\omega / 2 \chi}$ is the inverse thermal wavelength in the right wall of the vessel, $\chi$ is the temperature conductivity of it, $k=\omega / u_{2}$ defines the inverse wavelength of second sound.

Odd harmonics resonances of the heat flow appear and characteristics of these resonances depend on $h$. When $h$ is much less then the length of heat wave in the right wall of the vessel we obtain the expression for resonance of the heat flow:

$$
A(\omega)=\frac{Q_{0} u_{2}}{l_{2} \sqrt{\left(\omega-\omega_{0}\right)^{2}+\gamma^{2}}}
$$

Here $\omega_{0}$ is a resonance frequency and $\gamma$ is the width of the resonance peak:

$$
\gamma=\frac{u_{2}^{2}}{C_{V} \chi l_{2}} h
$$

Temperature odd harmonic resonances of unusual form appear (Fig. 4). When $h$ is much less then the length of heat wave in the right wall of the vessel we obtain the expression for resonance of the temperature: 

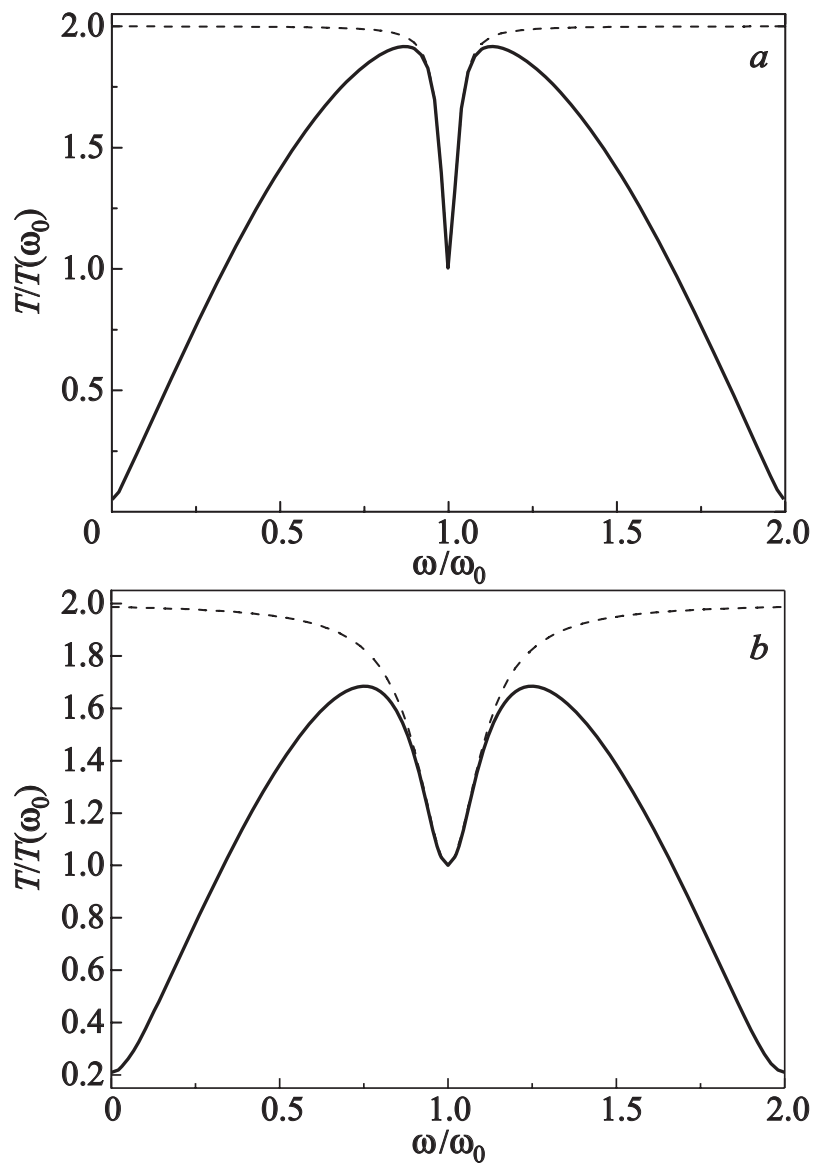

Fig. 4. An example of temperature resonance of unusual form with $\gamma=0.03 \omega_{0}(a), \gamma=0.13 \omega_{0}(b)$.

$$
A(\omega)=\frac{Q_{0}}{u_{2}} \sqrt{\frac{4\left(\omega-\omega_{0}\right)^{2}+\gamma^{2}}{\left(\omega-\omega_{0}\right)^{2}+\gamma^{2}}} .
$$

Such a behavior near a resonance frequency is much unexpected. The nature of this phenomenon is connected with the unusual type of the considered system. It consists of two layers which have different type of heat transfer acoustic and dissipative. This frequency dependence could be observed in future experiments. All the conditions under which such resonances can be observed are presented in this paper.

\section{Conclusions}

In conclusion we should note that the double-layer model of heat emission and propagation in helium is built. We have considered two double-layer systems: heater-helium and helium-detector. The model takes into account both second sound mode and thermal conductivity in superfluid helium and dissipative heat transfer in heater and the detector.

Resonance frequency and amplitude dependence on width of the heater and the detector was studied. The explicit expressions for temporal and spatial dependencies of temperature (14) and heat flow (17) were derived. In particular, these equations allow demonstrating the dependences of resonance frequency on the heater width (Fig. 3). Conditions, under which thermometer measures not temperature but heat flow, are determined and the expression (24) for the temperature in this case was obtained.

Unusual heat flow resonances behavior (33) is found out and experimental conditions under which such phenomenon may be observed are defined. Resonance frequency dependence on the heater length is found out. Influence of the right wall of the vessel on resonance characteristics is studied. Particularly, it was found, that the resonance width in helium can be determined not by dissipative properties of helium, but the dissipation in detector (32).

1. I.M. Khalatnikov, An Introduction to the Theory of Superfluidity, Redwood City, Addison-Wesley (1989).

2. V.P. Peshkov, J. Exp. Theor. Phys. 8, 16 (1946).

3. V.P. Peshkov, J. Exp. Theor. Phys. 10, 18 (1948).

4. A.S. Rybalko, Fiz. Nizk. Temp. 30, 1321 (2004) [Low Temp. Phys. 30, 994 (2004)].

5. K. Nemchenko and S. Rogova, QFS, 1-7 August, Grenoble (2010), p. 37.

6. K. Nemchenko and S. Rogova, International Conference for Young Scientists "Low Temperature Physics", 7-11 June, Kharkiv (2010), p. 105.

7. K. Nemchenko and S. Rogova, J. Low Temp. Phys. 150, 187 (2008).

8. K. Nemchenko and S. Rogova, J. Mol. Liq. 151, 9 (2010). 